\title{
Adapting LTE M-2-M Protocol Standard to Fulfil Latency Requirements for IEC 61850 G00SE Messages
}

\author{
Ali M. Allam, Mohamed Saied, Ihab Aly \\ Department of Electronic, Communication and Computer Engineering, Helwan University, Cairo, Egypt \\ Email: ali allam@h-eng.helwan.edu.eg, Mohamed.s.omar7788@gmail.com, ihabaly@ieee.org
}

Received 14 April 2015; accepted 9 May 2015; published 12 May 2015

Copyright (C) 2015 by authors and Scientific Research Publishing Inc.

This work is licensed under the Creative Commons Attribution International License (CC BY). http://creativecommons.org/licenses/by/4.0/

(c) () Open Access

\begin{abstract}
Generic Object Oriented Substation Event (GOOSE) Messages face a great challenge in its communication requirements especially in latency point of view. The appearance of the Long-Term Evolution (LTE) has opened a new way that can be used due to its great performance, compared with previous wireless technologies. In order to use LTE technology within the GOOSE message transfer, several points must be put into consideration, and additional requirements need to be within both LTE User Equipment (UE) side and network side. In this paper, we are going to adapt part of the LTE standards to fulfil the requirements of GOOSE messages which needs latency more than or equal to $20 \mathrm{~ms}$, using an experimental approach. The results show that LTE can satisfy the target requirements of latency within the acceptable Bit Error Rate (BER).
\end{abstract}

\section{Keywords}

M2M, LTE-A, G00SE Messages, Smart Grid

\section{Introduction}

Smart grid technologies are continuously developing, in order to improve the overall behaviour of the power network. This quick development faces great challenges towards its information gathering while transmitting the suppliers and consumer data. Smart grid system has different components with different communications requirements for each application.

GOOSE messages are a type of messages that defined in IEC 61850 [1] that transfer between intelligent substations to manipulate the performance and safety of substations, high-speed detection, isolation and restoration 
of faults on the whole distribution network. Some types of GOOSE messages need a critical latency that makes it use the Ethernet technique in order to save the time consumed in the network protocol stack upper layers, but after the approach to transmit these messages over a Wide Area Network (WAN), Ethernet can't be used because it's simply not a routable protocol [2].

LTE is the latest technology in wireless communication and categorized by its latency advantage compared with the previous wireless technologies [3]. The usage of LTE in GOOSE messages transmission will add several benefits as it will decrease the installation time for the communication environment, introduce a new solution for GOOSE messages transmission through WAN and also can be used as a backup solution for the current techniques.

Using LTE in smart grid system can be classified as Machine-2-Machine (M2M) communication, which is one of the biggest growing industries in the near future and it is a new breed to drive further enhancements of the LTE network.

Our contribution is adapting LTE standard that will enhance the LTE latency while sending GOOSE messages, by using the experimental approach for these adaptation and simulation approach to test the reliability of the suggested adaptation on the transmission GOOSE messages.

The organization of the next sections is as follows, in Section 2 it discusses the previous literatures, Section 3 contains information about the LTE network and its adaption for GOOSE messages requirements, Sections 4 and 5 contain the experimental work in LTE UE side, and the results and the conclusion are in Section 6.

\section{Related Work}

Synchrophasor communication for substations introduced in [4] and a comparison made between LTE, HSPA and VDSL. The results were made on a 1-way transmission, using packets of size 48 and 96 bytes, and almost in case of LTE the latency was less than 100 ms. Real-time scheduling in LTE for smart grids in [4] have offered a new scheduler technique in order to improve the latency of the assign the resources to the Smart Grid entities within LTE.

IEC 61850 GOOSE message over WAN presented in [2], Because of the new requirements that tends to transfer GOOSE messages within the WAN, to enhance the communication between substations, new techniques have been carried out in order to check the capability of using the internet with GOOSE messages. Using the internet appeared because of the Ethernet is not capable to be routed within the WAN and is not routable protocol. After trying Layer 2 tunnelling technique, it observed that the transmission time limit exceeded and this technique is not able to carry out the GOOSE messages [2].

The latency over LTE network discussed in [3], but several assumptions taken into consideration, as the processing time for Uplink and Downlink for UE, neglecting the standard that the UE have to wait $4 \mathrm{~ms}$ after receiving the grant from the network. It assumed that the UE side that the uplink processing time take 0.5 in layer 2 and another $0.5 \mathrm{~ms}$ in Layer 1.

In [5] [6] the authors have used WiMAX in GOOSE message application, the Latency of WiMAX network reached a range 30 - 50 ms. This latency has been achieved after making a unique "stand alone" mode in which the WiMAX equipment can support Layer 2, and able to propagate the message inside the same cell without the need of using centralized (Access Services Network gateway). The Latency achieved was acceptable, compared with GPRS network, but they did not compare these results with LTE.

\section{Adapting LTE to GOOSE Messages Requirements}

In order to adapt the LTE within GOOSE messages, the requirements of GOOSE have to be defined with LTE latency.

\subsection{GOOSE Messages Communication Requirements}

Standard IEC 61850-5 [1] introduced the communication requirements for functions and device models in the substations. Based on IEC 61850-5, there are 6 types of GOOSE messages with different communication requirements and performance classes.

Table 1 briefly defined the required latency for different types of GOOSE messages with their performance classes. 
Table 1. Latency requirements for GOOSE messages [1].

\begin{tabular}{ccc}
\hline Type & Class & Transmission time (ms) defined by trip time \\
\hline & P1 & 10 \\
Type 1A & P2 & 3 \\
& P3 & 3 \\
Type 1B & P1 & 100 \\
Type 2 & P2 & 20 \\
Type 3 & & 20 \\
& & 100 \\
Type 4 & P1 & 500 \\
& P2 & 10 \\
Type 5 & P3 & 3 \\
\hline
\end{tabular}

\subsection{LTE Latency}

LTE network latency for data transmission is named as User plane (U-Plane) latency, which is defined as the one-way transit time between a packet being available at the IP layer in either the User Equipment/Radio Access Network (UE/RAN) edge node or the availability of this packet at the IP layer in the RAN edge node/UE [3]. The reason of the U-plane latency acquired due to the time taken within the data path within the LTE network.

\subsubsection{Data-Path}

Figure 1 shows the equipment-2-equipment communication process, as used in our experiment, between the Phasor Measurement Unit (PMU), which represent the source and Phasor Data Concentrator (PDC) that represent the receiver within the LTE network.

The data path within the LTE network from source to destination have to follow a specific path, in which the UE request grant from the evolved NodeB (eNB) requesting a resource to transmit its data, once the eNB provide the UE with the requested resource the UE will send its data to the eNB. After that, the eNB will be forwarded directly the packet to the Serving Gateway (SGW), which will route the packet to its destination and forward it to the serving eNB. After that, the eNB will forward the packet to the destination UE.

Table 2 mentions the time taken by each process within the data path, before applying our suggested adaptive.

In order to maintain the latency of at least 20 ms to serve Type1B of class P2 and P3 messages an enhancement must take place at the UE side.

\subsubsection{UE Latency}

According to the standard 36.213 [8], once the UE receives a valid grant from the network in sub frame "n", it will transmit the data in sub frame " $n+4$ ", that means the transmission delay within UE in Frequency Division Duplex (FDD) mode is constant and equals to $4 \mathrm{~ms}$. The UE consume these $4 \mathrm{~ms}$ in two parts.

\section{(1) Layer 2/Layer 1 Processing Time}

The time taken by the UE processing time for handling the received data. In case of transmission of GOOSE messages it's one way transmission from the source to destination, that will reflect on the source no data to be handled in the downlink process, and the UE will handle the Up-Link (UL) data only.

(2) Timing Advance

Timing advance means that the network tells the UE to transmit the data a little bit earlier, depending on the distance between the UE and eNB. In the worst case that the distance between the UE and eNB is $100 \mathrm{Km}$ the 


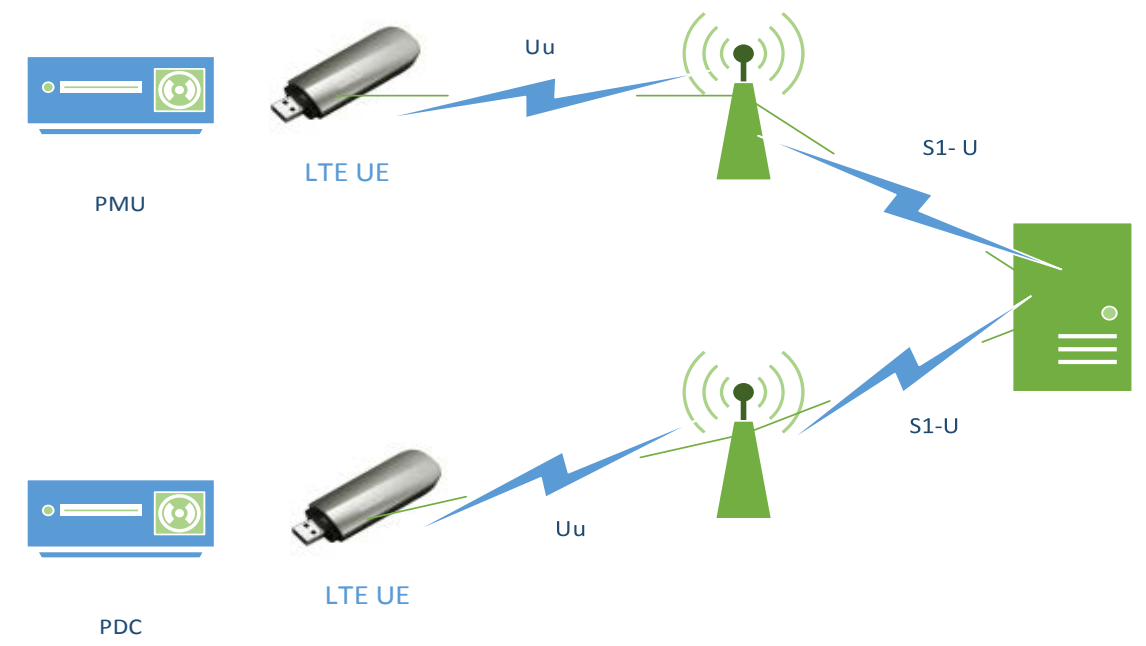

Figure 1. Diagram for PMU/PDC within LTE network.

Table 2. Overall latency within LTE network [7].

\begin{tabular}{cc} 
Process & Time in ms \\
UL Resource assignment & 8 \\
UE UL Data transmission processing Time & 4 \\
eNB Processing time & 3 \\
S1-U & 5 \\
SGW Processing Time & 5 \\
S1-U & 3 \\
eNB Processing time & 3 \\
UE DL Processing Time & 33 \\
Total Latency & 3 \\
\hline
\end{tabular}

timing advance will be 667 Microsecond [9].

\section{Suggested Adapted LTE Implementation}

A proposed change in standard 36.213 [8], to optimize the latency within LTE UE can take place, in which the LTE UE can send the required data in the next sub-frame and not to wait 4 sub frames that will be proved by implementing LTE UE Layer 2 and calculating its processing time.

This proposal appeared because of the GOOSE messages size that will not exceed 300 Byte [9], while each sub-frame in LTE can carry out more than $6 \mathrm{~K}$ bytes per sub frame.

The implementation will be carried out on uplink data of size 300 bytes which represent the actual size of GOOSE message [9], using Micro Controller embed LPC1768 based on the NXP LPC1768, ARM ${ }^{\circledR}$ Cortex $^{\text {TM }}$ M3 Core, with a 32-bit ARM Cortex-M3 core running at $96 \mathrm{MHz}$.

The implementation will take place on the edge of Layer 2 for LTE UE.

\subsection{Data Processing for Our Adaptation}

Once the MAC receives a valid grant from the network, it will indicate the Packet Data Convergence Protocol (PDCP) by this information. The PDCP sub layer will use 7-bit serial number (SN) length frame structure after that it will transfer the data from the upper layer to Radio Link Control (RLC) maintaining the PDCP serial number. The RLC sub layer will use unacknowledged mode, maintain the RLC header with Unacknowledged 
Mode Data Packet Data Unit (UMD PDU) SN and pass the RLC PDU to the MAC.

The MAC sub layer will deliver the data from the upper layer (RLC) to Layer 1, maintaining the MAC PDU header with the appropriate values of timing advance value indicating the physical layer to send its data in the next sub frame. To test the reliability of suggested adaptation, LTE network simulation was done, in order to prove the reliability of and its ability to be used within GOOSE messages from the point of view throughput and Bit error rate.

\subsection{LTE Network Simulation}

Using open source LTE Uplink level simulator from Vienna University [10] of Technology we were able to determine the Throughput of GOOSE messages over LTE network.

Additional changes added to the previously discussed simulation; by implementing Layer 2 to the simulator to transmit the required data of GOOSE message size in the next sub-frame directly once receive the grant from the network. Table 3 presents the simulation parameters. The result will be present and discusses in the next section.

\section{Results}

As observed in Figure 2, the processing time for 300 bytes of data in the uplink process for Layer 2 took 13 Microseconds.

In Figure 3, sending GOOSE message of package size 300 Byte, in each Transmission Time Interval (TTI) assigned to the UE, the UE throughput reached $3.5 \mathrm{Mbps}$, which is very acceptable compared with the capability of LTE to send hundreds of Mbps.

Figure 4 discusses the BER vs. SNR for UE within LTE network, we can see that the coded BER have significant improvement compared to un-coded, i.e. at BER $10^{-6}$ for un-coded is $16 \mathrm{~dB}$ while for coded is $22 \mathrm{~dB}$.

\section{Conclusions}

The UE has the ability to send GOOSE messages within size of 300 bytes of data in the next sub-frame after receiving the grant from the network and not to wait 4 milliseconds in FDD.

Applying these changes to the standards can enhance the responsiveness of the LTE UE and will reflect in decreasing the overall latency of the LTE network to make it able to carry our GOOSE messages within latency 20 ms network, as in Figure 5, which compare the latency performance of LTE after and before adaptation.

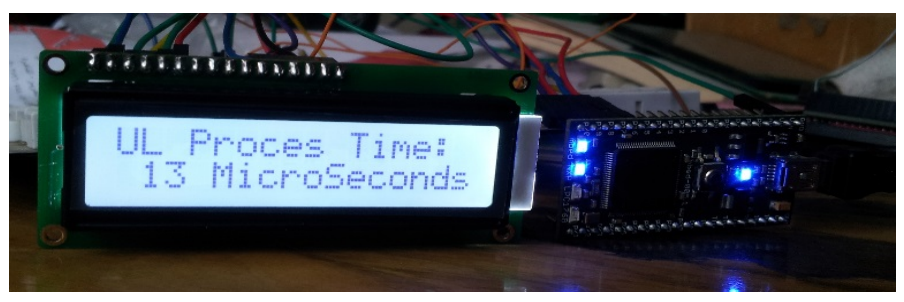

Figure 2. Layer 2 uplink processing time.

Table 3. Simulation parameters.

\begin{tabular}{cc}
\hline Parameter & Value \\
\hline Uplink frequency & $1.9 \mathrm{MHz}$ \\
Bandwidth & $1.4 \mathrm{MHz}$ \\
Number of TX/Rx antennas & Single Input Single Output (SISO) \\
Number of UEs & 1 UE \\
Speed of UE & Zero \\
Number of simulated sub frames & 10,000 \\
Scheduler setting & Static scheduler \\
\hline
\end{tabular}




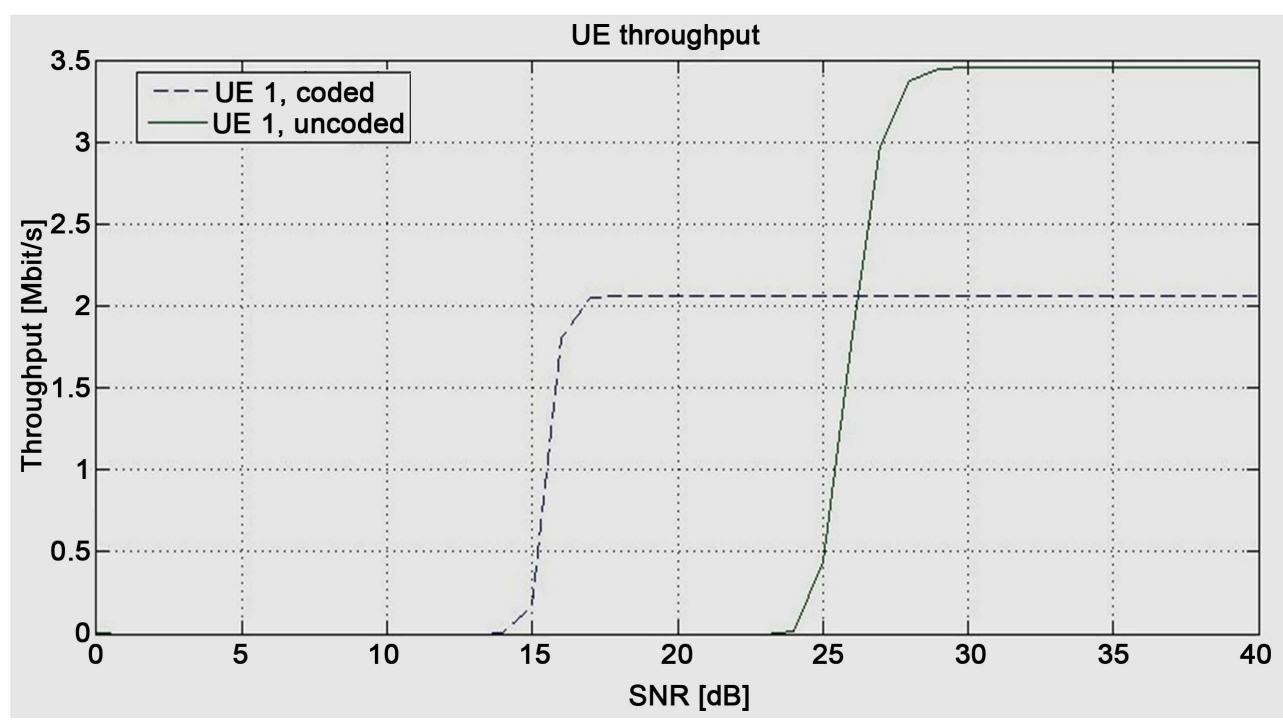

Figure 3. UE Throughput for transmitting 300 Byte in each TTI.

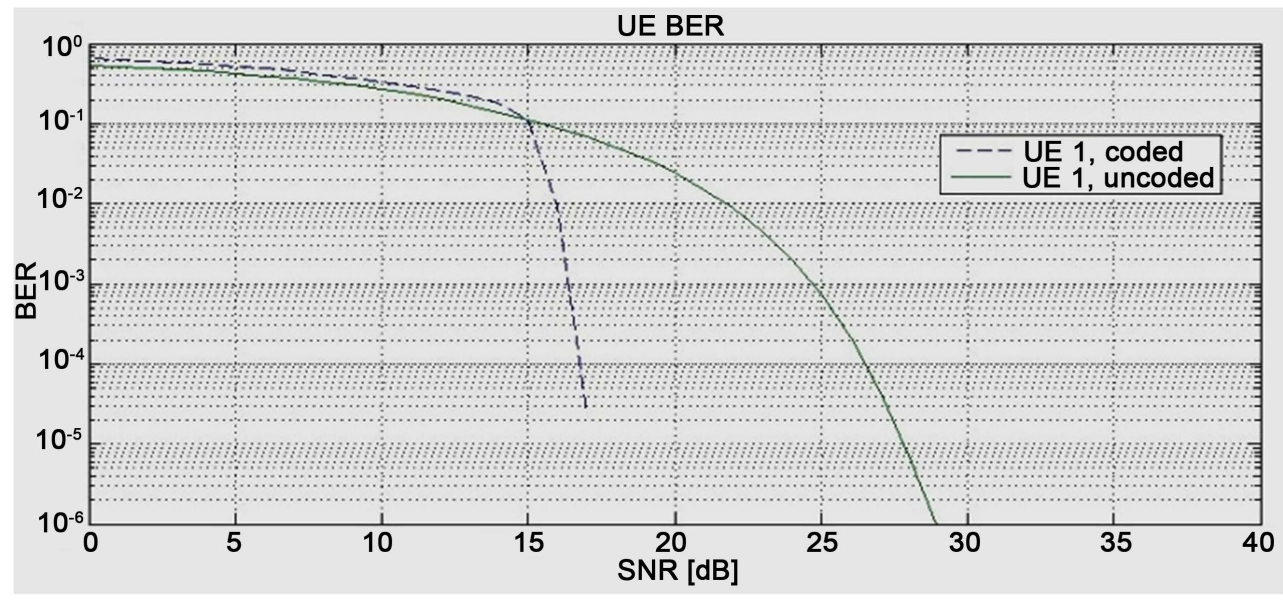

Figure 4. UE bit error-rate.

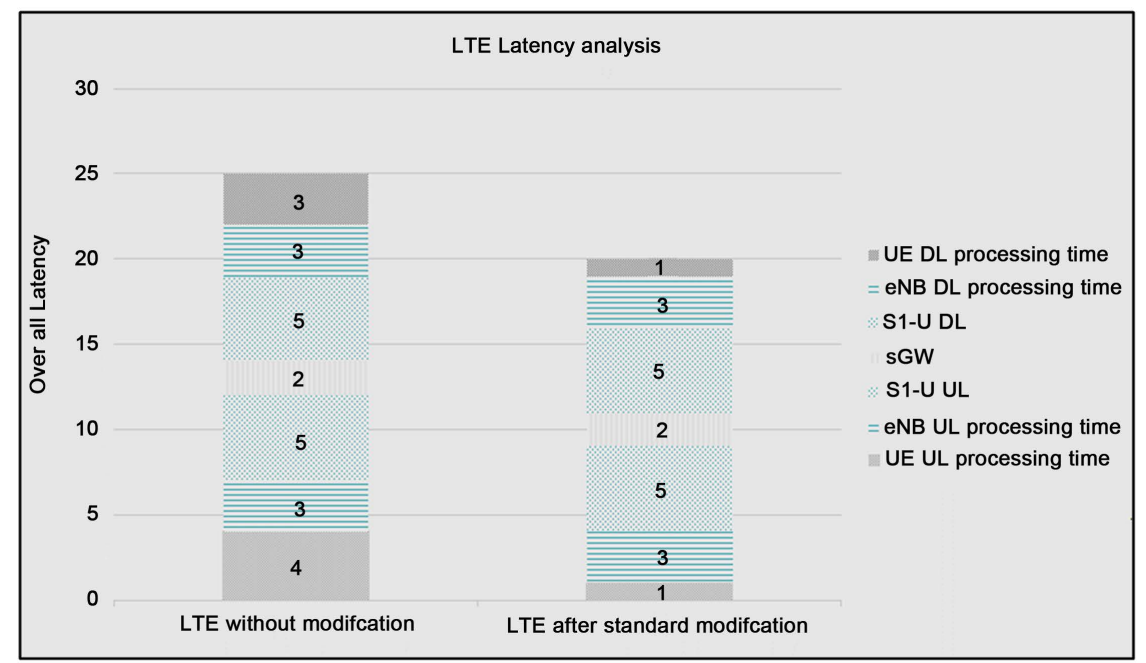

Figure 5. Overall LTE latency after applying the UE changes. 
LTE network is reliable to handle GOOSE messages with acceptable throughput and BER after applying suggested adaptation. Sending the required data in the next sub frame will not affect either the throughput or the BER, this is due to that the UE can be sent in each sub frame.

\section{References}

[1] Baigent, D., Adamiak, M. and Mackiewicz, R. (2003) IEC 61850-5, Communication Networks and Systems in Substations-Part 5: Communication Requirements for Functions and Device Models.

[2] de Oliveira, C.H.R. and Bowen, A.P. (2012) IEC 61850 GOOSE Message over WAN. The 2012 International Conference on Wireless Networks (ICWN'12), Las Vegas.

[3] Blajić, T., Nogulić, D. and Družijanić, M. (2006) Latency Improvements in 3G Long Term Evolution. Mipro CTI.

[4] Zseby, T., Fabini, J. and Rani, D. (2014) Synchrophasor Communication. e\&iElektrotechnik und Informationstechnik, 131, 8-13.

[5] Xu, Y.Z. and Fischione, C. (2012) Real-Time Scheduling in Lte for Smart Grids. 5th International Symposium on Communications Control and Signal Processing, Rome, 2-4 May 2012, 1-6. http://dx.doi.org/10.1109/ISCCSP.2012.6217872

[6] Maciej, G., Lee, L. and Jim, M. (2011) IEC 61850 Goose over Wimax for Fast Isolation and Restoration of Faults in Distribution Networks. 21st International Conference on Electricity Distribution, Frankfurt, 6-9 June 2011, paper 1322.

[7] Mohan, S., Kapoor, R. and Mohanty, B. (2011) Latency in HSPA Data Networks. Qualcomm, Tech. Rep.

[8] 3GPP TS 36.213 V12.0.0 (2013-12) Technical Specification 3rd Generation Partnership Project, Technical Specification Group Radio Access Network, Evolved Universal Terrestrial Radio Access (E-UTRA), Physical Layer Procedures (Release 12).

[9] de Oliveira1, J.C., Varella, W.A., Marques, A.E. and Forster, G. (2007) Real Time Application Using Multicast Ethernet in Power Substation Automation According to IEC61850. White Paper, PAC World Journal.

[10] Vienna Simulation, LTE Uplink Link Level Simulator. http://www.nt.tuwien.ac.at/research/mobile-communications/lte-uplink-link-level-simulator/ 